\title{
The 'Perfect Storm' and Acute Coronary Syndrome Onset: Do Psychosocial Factors Play a Role?
}

\author{
Matthew M. Burg, PhD, Donald Edmondson, PhD, Daichi Shimbo, MD, Jonathan Shaffer, \\ $\mathrm{PhD}$, Ian M. Kronish, MD, MPH, William Whang, MD, Carmela Alcántara, PhD, Joseph E. \\ Schwartz, PhD, Paul Muntner, PhD, and Karina W. Davidson, PhD \\ Center for Cardiovascular Behavioral Health, Department of Medicine, Columbia University
}

\section{Abstract}

The revolution in cardiac care over the past two decades, characterized by emergent revascularization, drug eluting stents, anti-platelet medications, and advanced imaging has had little impact on overall ACS recurrence, or ACS prevention. The "Perfect Storm" refers to a confluence of events and processes, including atherosclerotic plaque, coronary flow dynamics, hemostatic and fibrinolytic function, metabolic and inflammatory conditions, neurohormonal dysregulation, and environmental events that give rise to, and result in an ACS event. In this article we illustrate the limits of the traditional main effect research model, giving a brief description of the current state of knowledge regarding the development of atherosclerotic plaque and the rupturing of these plaques that defines an ACS event. We then apply the Perfect Storm conceptualization to describe a program of research concerning a psychosocial vulnerability factor that contributes to increased risk of recurrent ACS and early mortality, and that has defied our efforts to identify underlying pathophysiology and successfully mount efforts to fully mitigate this risk.

\begin{abstract}
There is a "Perfect Storm" coming. Although this metaphor has been used to warn us about the ongoing U.S. fiscal crisis and about impending weather events, it is also a potentially useful metaphor when applied to the occurrence of an acute coronary syndrome (ACS). 1,2 The "Perfect Storm" as a conceptual model applied to ACS proposes to us that ACS events are not caused by the presence of a single, or even a number of specific coronary heart disease (CHD) risk factors, but rather results from an unfortunate confluence involving any of several stable underlying CHD vulnerability factors and the co-occurrence of any of several situational or environmental events that acutely activate critical physiological processes, thereby contributing overall to a period of increased risk. ${ }^{3}$ Thus the "Perfect" ACS storm is defined as the joint presence of many co-occurring pathophysiologic processes that lead to a clinical event. This Perfect Storm will happen for more than 1 million American adults each year. Many of these individuals will join the more than 17 million living American adults who have survived an ACS but continue to suffer from the cardiovascular damage remaining after the ACS event. ${ }^{4}$ Understanding how the Perfect Storm model aids in the investigation of this large public health problem is an exciting
\end{abstract}

(C) 2013 Elsevier Inc. All rights reserved.

Correspondence should be addressed to: Karina W. Davidson, Center for Behavioral Cardiovascular Health, Department of Medicine, Columbia University Medical Center, PH9, Room 9-319, 622 W. 168th Street, New York, NY 10032, kd2124@ columbia.edu.

Conflict of interest statement: None to report

Publisher's Disclaimer: This is a PDF file of an unedited manuscript that has been accepted for publication. As a service to our customers we are providing this early version of the manuscript. The manuscript will undergo copyediting, typesetting, and review of the resulting proof before it is published in its final citable form. Please note that during the production process errors may be discovered which could affect the content, and all legal disclaimers that apply to the journal pertain. 
venture that may provide new insights into how to prevent ACS, and into the management of CHD for those already suffering from the physiological consequences of an ACS.

\section{The Perfect Storm Metaphor and Psychosocial Risk Factors}

In this article we apply the Perfect Storm framework to the study of psychosocial risk for cardiovascular disease. We start by illustrating the limits of the traditional research strategy that identifies a risk factor in isolation and by giving a brief description of the current state of knowledge regarding the development of CHD and the vulnerable plaque concept that underlies the presentation of an ACS event. We then consider how a research agenda within a Perfect Storm framework would differ from the traditional approach that has guided investigation and contributed to our understanding of ACS to date. We use this as an impetus to then describe the application of the Perfect Storm conceptualization in the development of a program of research concerning a psychosocial vulnerability factor that contributes to increased risk of recurrent ACS and early mortality, and that has defied our efforts to identify underlying pathophysiology and successfully mount efforts to fully mitigate this risk.

\section{Coronary Atherosclerosis and ACS-A View of the Traditional Research Strategy}

There continues to be a revolution in cardiac care that began two decades ago. This revolution is epitomized by the emergence of new intervention and imaging technologies that have improved cardiac mortality outcomes substantially, at least for those patients who present with ST-elevation myocardial infarction. Yet they have had little impact on overall ACS recurrence, ${ }^{5,6}$ nor have they improved our ability to prevent ACS.

Coronary atherosclerosis is a progressive disease that initiates as early as the childhood and adolescent years, and depending on a range of genetic, behavioral, and environmental factors, progresses into adulthood, such that upwards of $80 \%$ of middle-aged adults in developed nations have evidence of coronary artery disease (CAD). Yet the annual incidence of ACS in the United States for people over age 40 is less than $1 \% .^{7}$ Thus, merely the presence of CAD is not sufficient for ACS and other factors must be involved. Research efforts have therefore been directed at identifying the factors that lead from the development and progression of atherosclerotic plaque in the coronary arteries to the catastrophic rupture of these plaques and the occurrence of ACS. As this research has progressed it has become apparent that, 1) most ACS events are caused by plaque disruption with subsequent occlusive platelet-thrombus formation, but 2) the converse is not true - e.g., most disrupted coronary plaques with platelet-thrombus formation do not lead to ACS event onset. ${ }^{8}$ Pathology and clinical studies have shown that plaque rupture and non-occlusive thrombus formation are frequently present in the absence of clinical ACS symptoms. Therefore, an ACS event is not a necessary consequence of coronary plaque disruption, and while plaque disruption may be a necessary cause of ACS, it is by no means sufficient.

\section{Plaque Rupture and Thrombosis, and the Search for the Vulnerable Plaque}

Today, research continues to focus on the factors that will help practitioners identify the vulnerable plaque before it ruptures, so as to prevent ACS. Factors such as morphology and genetics, have been most frequently studied in isolation. Researchers have thus given less consideration - for example - to the properties of blood that underlie the thrombosis precipitated by plaque rupture, the properties of coronary vasculature that support or resist thrombus formation, or any of several other features of the individual that might be implicated in the complex cascade that defines an ACS event. This type of investigation is 
sometimes referred to by methodologists as a 'main effects' model. This approach to science implicitly assumes that the one factor under investigation - in this case plaque vulnerability - is both necessary and sufficient to cause the outcome - an ACS event. This "reductionist disease model"9 has taken the methodological tact of dissecting systems into their constituent parts, and it has been very effective in providing insight into many biological and chemical components of living processes. ${ }^{10}$ This approach has however, reached its limit. Biological systems are exceedingly complex and have emergent properties that cannot be explained, or even predicted, by studying their individual parts. ${ }^{10}$ While this simplifying methodological approach has proven invaluable in many human disease areas including CHD - it can lead to oversimplifications that limit investigation, understanding, and the development of new treatments.

\section{The Perfect Storm Conceptual Model: Implications for CHD Classic Risk Factors and Pathophysiologic Processes}

Recently, Arbab-Zadeh and colleagues ${ }^{3}$ in an elegant review of the pathophysiology underlying ACS events, used the term "Perfect Storm" to describe the confluence of events and processes that give rise to, and result in an ACS (see Table 1). The factors they list that are associated with increased ACS event risk are categorized according to characteristics of atherosclerotic plaque (e.g., endothelial dysfunction, plaque composition/location/burden/ configuration,); coronary flow dynamics (e.g., blood viscosity, shear stress, vascular tone/ reactivity); instrinsic hemostatic and fibrinolytic function (e.g., platelet function); metabolic and inflammatory conditions (e.g., diabetes, obesity); neurohormonal dysregulation (e.g., depression, catecholamine surge, autonomic dysfunction); and environmental factors and triggers (e.g., stress). While they state that it remains to be fully determined whether a particular factor plays a role specifically in the development and progression of atherosclerosis, in the frequency of plaque alterations, or in the promotion of thrombotic vascular occlusion, they emphasize that it may be a combination of these effects that underlies their linkage to ACS risk. Similarly, it is understood that many of these factors serve to influence others. For example, psychological stress will dynamically influence autonomic function, resulting in an increase in sympathetic activity, as evidenced by a catecholamine surge. This will in turn increase shear stress within the coronary circulation, particularly at arterial branching points, which are often the site of atherosclerotic lesions. Lastly, since many of these factors evidence a circadian variability, "timing" may be an essential ingredient of the Perfect Storm. Thus, the catecholamine surge that is typically seen in the early part of the day may serve as an ACS trigger, depending on the plaque, vascular, and platelet conditions that by happenstance are co-occurring at that time on a given day. Yet, on a preceding day or a subsequent day, the conditions of the vasculature, plaque, and/ or blood may not be sufficient for an ACS, given the absence of that catecholamine surge. Similarly, factors such as exposure to pollutants (e.g., tobacco smoke) or stress, or post prandial effects, can influence ACS risk throughout a given day, by impacting on several factors including endothelial function, but again also depending on the transient conditions of several other critical aspects of the Perfect Storm.

The complexity of the various factors and their inter-relationship in the progression from coronary atherosclerosis to ACS leads the authors to state that, "one may establish certain probabilities for plaque rupture/erosion and occlusive thrombus-favorable conditions to coincide, but a substantial residual component of uncertainty will remain". While this may be somewhat disheartening to those who share an interest in a future defined by a substantially reduced universal ACS risk, all is not lost. The Perfect Storm concept provides a potentially highly useful framework within which to articulate testable hypotheses that begin to include the wide array of pathophysiological processes underlying and contributing to atherosclerosis and ACS risk. Indeed, this conceptualization may be a critical basis for 
planning and executing future research studies that will provide greater insights into the ACS phenomenon. An approach such as this could contribute to the development and testing of improved primary and secondary prevention strategies that do not rely on an overly simplistic "main effects model", and thereby result in better overall patient management. In the next Section we demonstrate the utilization of this model in the articulation of a research program focusing on the confluence of two Perfect Storm factors - depression and exposure to acute stress- that each contribute significantly and independently to ACS recurrence and mortality risk, and that when present together, may better aid our investigation of the underlying pathophysiology of their ACS recurrence risk.

\section{Depression and Risk of Recurrent ACS and Mortality}

As reviewed in greater detail elsewhere in this volume, epidemiological studies have demonstrated convincingly that ACS patients with depression are at greater risk for ACS recurrence and mortality compared to their non-depressed counterparts. ${ }^{1-14}$ The hazard ratio associated with depressive symptoms is 1.80 (95\% CI: 1.46-2.51) for all-cause mortality, ${ }^{15}$ and 1.95 (95\% CI: 1.33-2.85) for myocardial infarction (MI) recurrence. ${ }^{16} \mathrm{~A}$ recent meta-analysis shows that depressive symptoms continue to strongly identify ACS patients at mortality risk [relative risk: 3.41 (95\% CI: $2.19-5.31 .{ }^{15}$ Depressive symptoms are on par with more conventional ACS recurrence prognostic factors for predicting death and ACS recurrence. This risk continues to be observed in the most recent studies and metaanalyses, despite the continual improvement in cardiology interventions, medications, and care. ${ }^{17-21}$

\section{Depression and Post-ACS Recurrence/Mortality Risk: Mechanism(s) of Action}

Many biological and behavioral mechanisms may underlie the depression-ACS recurrence and mortality link, and a number of these have been examined systematically. ${ }^{22}$ Proposed biological mechanisms include greater risk of arrhythmogenesis - in part due to alterations in autonomic regulation ${ }^{23,24}$ and25,26 impaired coronary flow reserve and associated vascular factors that increase risk of myocardial ischemia. ${ }^{27,28}$ Proposed behavioral mechanisms include adherence to prescribed medications ${ }^{29,30}$ and engagement in secondary prevention behavioral recommendations such as avoiding a sedentary lifestyle and receiving sufficient sleep. ${ }^{31}$ Inconsistent findings have been reported for each of these proposed mechanisms, however. ${ }^{15}$ Thus, while it is now widely accepted that depressive symptoms after ACS predict poorer medical prognosis, there remains a gap in our knowledge about which of these proposed mechanisms best account for this association. Without this knowledge, we cannot adequately target mechanisms in clinical trials so as to reduce this risk.

\section{Depression and Post-ACS Recurrence/Mortality Risk: A Perfect Storm?}

Most research on depression related post-ACS recurrence and mortality risk has been conducted with an implicit "main effects model" of how causal mechanism(s) responsible for this risk would operate. This model can be described according to the following three implicit assumptions: (1) depressive symptoms in ACS patients would directly disrupt the proposed mechanism at all times; (2) the presence of some additional Perfect Storm factor would not be required to reveal the presence of the disrupted mechanism, and (3) the presence of some additional Perfect Storm factor would not differentially affect ACS patients with depressive symptoms compared to those without depressive symptoms. These three implicit assumptions have led us to use a simple between-subject design, comparing depressed and nondepressed ACS patients, and looking for differences in the mechanism that is hypothesized to underlie the depression related risk. When evidence has been 
contradictory, we have assumed that we have not yet identified the responsible mechanism(s). We propose instead that it is time to abandon the 'main effects' approach for understanding this depression associated risk, and consider the benefits of the more complex Perfect Storm conceptualization.

Historically, the study of psychosocial vulnerabilities for ACS such as depression, anxiety, hostility, and post-traumatic stress disorder has also followed the scientific tradition of employing the 'main effects' or reductionist model of science, and have frequently studied these in isolation of each other, and even in isolation of the classic CHD risk factors. ${ }^{15}$ Suls and Bunde pointed out the limits of this approach and suggested that we needed more complex models of the association of emotion and CHD in order to gain a better understanding of the role that emotions play in the etiology and course of CHD, and in the triggering of an ACS. ${ }^{32}$ Indeed, the consideration of two factors at the same time, such as the co-occurrence of a chronic angry temperament and getting angry in some specific situation, may be required to identify emotional factors that result in defibrillator shockterminated arrhythmias. ${ }^{33}$

\section{The Perfect Storm: A Model for a Program of Research}

How might we apply the Perfect Storm conceptualization to the study of mechanisms linking a Perfect Storm factor such as depression to post-ACS recurrence and mortality risk? We propose a model that, as with the Perfect Storm, describes a confluence of factors to describe the resulting risk of ACS recurrence and mortality. Within this Model, we test the effects of a confluence of both depression and acute stress on the activation of four distinct pathways that have each been observed in relation to depression, and that each have been linked to post-ACS medical prognosis. These four pathways are, 1) Blood - or thrombogenicity; 2) Coronary Perfusion - and related vascular factors; 2) Arrhythmia - and related autonomic factors; and 4) Behavior - broadly including physical activity, medication adherence, and sleep (see Figure 1). We thus provide a challenge to the traditional efforts directed toward elucidating the depression related ACS recurrence/mortality risk. Indeed, just as regional wall abnormalities and myocardial perfusion defects are identified by echocardiography and myocardial perfusion imaging only under exercise or pharmacologic stress, and these defects will remain undetected if stress exposure is not utilized, it may be that we have to 'stress' the depressed CHD patient to reveal the mechanisms responsible for depression related risk.

\section{Depression and Thrombogenicity}

Several small case-control studies have demonstrated the existence of platelet hyperreactivity in apparently healthy, depressed patients, ${ }^{25,26,34}$ though evidence of exaggerated platelet reactivity in depressed patients with CAD is limited. Laghrissi-Thode et $\mathrm{al}^{35}$ described higher platelet factor 4 (PF4) and $\beta$-thromboglobulin (BTG), markers of platelet aggregation, in depressed vs. non-depressed CAD patients. Gehi et al however, found no association between depression and either PF4 or BTG in CAD patients who were withdrawn from ASA for 7 days. ${ }^{36}$ Using pooled data from 3 studies of ACS patients, Serebruany et $\mathrm{al}^{37}$ found that depressed ACS patients exhibited higher levels of PF4, BTG, and platelet/endothelial cell adhesion molecule- 1 when compared to non-depressed ACS patients without depression.

There is also evidence that acute psychological stress increases the level of platelet aggregation. A systematic review by Thrall et al ${ }^{38}$ concluded that acute psychological stress is associated with increases in platelet aggregation, and suggested that these increases may have the potential of triggering ACS events. Several studies have shown that a mental stress task increases ADP-induced platelet aggregation in apparently healthy individuals and in 
patients with CAD. The data demonstrating an effect of a mental stress task on platelet aggregation are most consistent with immediate post-task measurements. Some studies have also shown effects on platelet aggregation at 30 and 60 minutes into recovery after a mental stress task. Although a few studies have indicated that basal levels of platelet aggregation are reduced by antiplatelet agents, these agents do not blunt mental stress-induced increases in platelet aggregation.

After an ACS event, when disrupted coronary plaque(s) are healing, it is plausible that depressed vs. non-depressed patients are at risk for recurrent MI/mortality because they have a greater increase in platelet aggregation in response to stress. In depressed patients, an exaggerated platelet-thrombogenesis response to psychological stress may induce occlusive thrombus formation leading to another ACS event, thus demonstrating that depression and stress together may create a Perfect Storm. The testing of this overall scenario - a comparison of depressed and non-depressed post-ACS patients in their response to psychological stress, preferably under controlled laboratory conditions, and examination of changes in key measures of thrombogenicity across resting and stress conditions represents one vehicle for implementation of a Perfect Storm conceptualization.

\section{Depression and Coronary Perfusion}

Among the "Perfect Storm's" upstream physiological processes identified as critical to the expression of an ACS event are endothelial (dys)function and vascular reactivity, as contributors to perturbations - or dysregulation - in dynamic myocardial blood flow. Endothelial dysfunction, seen in the earliest stages of CHD, is marked by a paradoxical or inappropriate vasoconstriction. This also has been observed among otherwise healthy individuals with depression. ${ }^{39}$ It has also been observed in response to mental stress among healthy non-depressed individuals, with blockade of endothelin-1 (ET-1), the most potent vaso-active protein, mitigating this effect. ${ }^{40}$ We have also observed that stable CHD patients with elevated depression symptoms demonstrate a level of ET-1 previously linked to early recurrent ACS. ${ }^{27}$

Since the description in the 1970s of myocardial ischemia provoked by mental and emotional stress, ${ }^{41}$ research has consistently shown that approximately half of patients with stable coronary artery disease (CAD) evidence this mental stress provoked myocardial ischemia (MSI) on myocardial perfusion imaging (see Figure 2) despite optimal medical management. ${ }^{42}$ We and others have shown a 2.4-3.0 fold increased independent risk of myocardial infarction over 1-4 years for patients demonstrating MSI, ${ }^{43-45}$ and a 3.0 risk ratio of 5-year mortality. ${ }^{46}$

The model of CAD that guides disease management focuses on the identification and mitigation of obstructive atherosclerotic plaque in the large coronary vessels. In contrast to this traditional model whereby ischemia occurs as a function of a static occlusion in the large coronary vessels that limit blood flow, mental and emotional stress ${ }^{41}$ induces temporal heterogeneity of myocardial perfusion. Yeung et $\mathrm{al}^{47}$ demonstrated that mental stress provokes a paradoxical epicardial vasoconstriction at the site of both obstructive and nonobstructive plaques, and a consequent reduction in myocardial blood flow, though whether this effect was due to epicardial vasoconstriction vs. microvascular dysfunction could not be ascertained. Using positron emission tomography we observed that during mental stress, coronary flow reserve (CFR) - the ability of the microvascular bed to dilate in response to stimulation and thereby augment coronary blood flow according to the needs of myocardial tissue - was lower in regions served by vessels without significant epicardial stenosis than in regions served by vessels with significant stenosis. Furthermore, while coronary resistance fell during mental stress in regions served by vessels with significant stenosis, coronary 
resistance increased in regions served by vessels without significant stenosis. ${ }^{48}$ This attenuation in myocardial blood flow during mental stress in regions without significant stenosis along with the increase in resistance, implicates the coronary microvascular bed in the pathophysiology of MSI. How might depression further contribute to this apparent dysregulation?

In one small study, the relationship of depression to MSI risk was tested in a heterogeneous sample of patients with coronary disease. ${ }^{49}$ A curvilinear relationship was observed, such that patients with mild to moderately severe symptoms of depression were at 2 -fold increased risk of MSI, while those with more severe symptoms of depression were not. The sample size was relatively small, the measure of depression has not been widely linked to ACS recurrence/mortality risk, and the marker of ischemia - wall motion abnormality - is less sensitive/specific than myocardial perfusion. ${ }^{48}$ Depression, myocardial perfusion, and coronary flow reserve in response to adenosine has been the focus of one small study ${ }^{28}$ of male twin pairs from the Twins Heart Study. Not surprisingly given that the sample was not selected for having known CAD disease, less than $10 \%$ of the sample demonstrated a significant myocardial perfusion abnormality with pharmacologic stress, and major depression was not a predictor of this outcome. This study did however, find greater impairment in CFR in dizygotic twins with a major depression diagnosis vs. their nondepressed control twins.

Both depression and acute stress are associated with perturbations in vascular processes endothelial function, myocardial perfusion, and CFR - that are part of the Perfect Storm articulated by Arbab-Zadeh and colleagues. As with thrombogenicity, the testing of this overall scenario - a comparison of depression and non-depressed patients after ACS, exposure to psychological stress, and examination of effects on regional myocardial perfusion and coronary flow reserve - represents a second vehicle for implementation of a Perfect Storm conceptualization.

\section{Depression and Arrhythmogenesis}

Most ACS patients present to the emergency department (ED), ${ }^{50}$ and during their stay it can often become overcrowded and chaotic. ${ }^{51}$ Admission to an ED when in the throes of an ACS is a stressful experience, and when that ED is overcrowded, it is exceedingly stressful. ${ }^{21,52} \mathrm{ED}$ crowding varies greatly through the course of a single day in most hospitals throughout the US, ${ }^{17,19,53}$ and is associated with greater in-hospital ${ }^{17,20} 30$-day, and 1-year post-ACS mortality. ACS patients treated during times when the ED is most crowded are 3 to 5 times more likely to experience subsequent in-hospital MI than those treated during less crowded times and are at significantly greater subsequent $\mathrm{MI} /$ mortality risk. ${ }^{18}$

Might the stress of the ED also be arrhythmogenic? From epidemiologic studies it has been shown that there is an increase in the incidence of sudden death associated with emotionally devastating disasters such as earthquake and war, ${ }^{54,55}$ and ventricular arrhythmias, one possible link between stress and sudden death, increased in the emotional weeks following the destruction of the World Trade Center in $2001 .{ }^{56}$ Strong emotion in response to real life stress can spontaneously trigger ventricular arrhythmias in patients with ICDs, ${ }^{57}$ and these arrhythmias are polymorphic, ${ }^{58}$ suggesting that they are potentially lethal. Further, psychological stress has been found to facilitate the induction, and increase the rate of ventricular tachycardia, and makes the termination of these arrhythmias more difficult, effects that are correlated with increases in circulating levels of epinephrine and norepinephrine. ${ }^{59}$ Further, ventricular tachycardia and sudden death occur more frequently in the morning hours, ${ }^{60}$ the time of peak catecholamine levels and lowest vagal tone. From 
this research we can see that among the effects of stress are a change in autonomic balance greater sympathetic activity as characterized by an increase in circulating catecholamines, and a reduction in cardiac vagal control as characterized by lower heart rate variability.

As with thrombogenicity and myocardial perfusion, the scenario of stress in the ED has features that are consistent with the Perfect Storm conceptualization of ACS, and ventricular arrhythmias during time in the ED might demonstrate this for at risk individuals. How might this be utilized to understand the contribution of depression to ACS recurrence and mortality risk? In a secondary analysis of data from a large cohort study of ACS patients, researchers found that those who report both depression and exposure to high stress were at significantly greater risk for MI recurrence or mortality. It is also known - as discussed in another article in this issue - that one feature of depression is autonomic dysregulation, characterized by a general increase in sympathetic activity and a reduction in cardiac vagal control, a scenario that could increase risk of ventricular arrhythmia. Thus both depression and acute stress are associated with the autonomic or neurohormonal effects that are part of the Perfect Storm articulated by Arbab-Zadeh and colleagues. ${ }^{3}$ The testing of this overall scenario in an effort to understand the depression related ACS recurrence and mortality risk could be accomplished by examining the effects of ED crowding on autonomic indices and incidence of ventricular arrhythmia, and determining whether depressed ACS patients admitted to the ED when it is most crowded and stressful evidence greater disruption in autonomic function and a greater number of ventricular arrhythmias. Such a "naturalistic" undertaking, where the stress is "real world" and is likely to vary throughout a patient's ED stay provides an elegance not otherwise provided for, and represents a third vehicle for implementation of a Perfect Storm conceptualization.

\section{Depression and Cardiovascular Risk Behaviors}

The last decade has been marked by significant improvements in the medical care of patients with cardiovascular disease, yet behavioral factors continue to account for a substantial proportion of the risk of MI recurrence and premature mortality. Indeed, behavioral mechanisms such as sedentary lifestyle may bear greater responsibility for the incidence of CHD than biological mechanisms. ${ }^{61}$ Similarly, a failure to adhere to recommended health behaviors after ACS - including the initiation of an exercise program and the taking of prescribed medications - contributes substantially to risk for recurrent ACS and early mortality. ${ }^{30,62-64}$ In fact, it is estimated that improving health behaviors could reduce CHD risk and the associated public health burden by up to $40 \% .{ }^{65}$

While it is widely accepted that depressive symptoms after ACS predict poorer medical prognosis, there remains a gap in our knowledge about the contribution of behavioral mechanisms to this association, and which of several such mechanisms best explain this association. Without this knowledge, we cannot adequately target mechanisms to reduce this risk.

Among potential behavioral mechanisms is medication adherence. It has been shown that in the weeks after suffering the distress of an ACS, depressed patients have lower medication adherence compared to non-depressed patients. ${ }^{63,66}$ Other studies however, have shown no differences in medication adherence between depressed and non-depressed patients, whether with ${ }^{67}$ or without ${ }^{68}$ comorbid CHD. Yet other studies of medication adherence show it to be a dynamic behavior that varies over time. ${ }^{69,70}$ The precise circumstances that contribute to poorer adherence at one time and better adherence at another time remain to be identified. It is possible that these inconsistent findings are driven by differences in study design that lead to different circumstances during which medication adherence was measured. 
Physical activity may be another important behavioral mechanism that accounts for much of the excess cardiovascular risk associated with depression. ${ }^{71}$ For example, it is well known that depressed post-ACS patients attend cardiac rehabilitation less frequently, ${ }^{72}$ exercise less, ${ }^{71}$ and report less physical activity overall, than do non-depressed post-ACS patients. ${ }^{31}$ Furthermore, in healthy adolescents, reduced physical activity predicts onset of depression. ${ }^{73}$ It is currently thought that physical inactivity predisposes one to depression, and that depression predisposes one to even more physical inactivity, creating a forward feed cycle that would be particularly harmful for recent ACS patients. ${ }^{74,75}$ Moreover, although there is evidence that exercise interventions can be efficacious for treating depression, ${ }^{76,77}$ it remains unknown if treating depression by exercise would reduce the depression-related ACS recurrence and mortality risk.

As described earlier, most research examining behavioral mechanisms of cardiovascular risk has been conducted with implicit assumptions characterized by a main effects model, such that if the depression-recurrence/mortality risk could be attributed to behavioral mechanisms, these mechanisms would always be observed in depressed but not in nondepressed post-ACS patients, and acute stress would have no differential effect on the behavioral mechanism. Yet, emerging research now shows that exposure to acute stress adversely impacts good health behavior practices. ${ }^{78}$ There is also emerging evidence in nonCHD patients that stress can be especially deleterious to health behaviors among patients with underlying depression. ${ }^{79}$ Depressed patients under acute stress might experience greater difficulty in self-regulation and thus fail to use cognitive, emotional, or behavioral resources to achieve desired health behaviour outcomes. This could furthermore increase their propensity to engage in adverse health behaviors. ${ }^{80}$

Again, the Perfect Storm conceptualization provides a rich context within which to develop an innovative research design so as to elucidate the contribution of behavior to the depression related ACS recurrence and mortality risk. This could be tested by examining the effects of real life stress on the conduct of specific health behaviors among post-ACS patients, and determining whether those with vs. without depression are more susceptible to the effects of stress. As part of standard cardiologic care, patients are often scheduled for "staged" percutaneous interventions. These events represent an excellent example of a standardized real life stressor that can be used to test the hypothesis that the confluence of stress and depression affects a patient's engagement in critical health behaviors. First, even though these represent planned rather than emergent procedures, the experience of undergoing a percutaneous intervention is likely perceived as very stressful by patients as they require spending a day in the hospital, which markedly disrupts their daily routine including their diet, sleep schedules, work, and social networks. Patients are also immediately immersed in an unfamiliar medical system where they receive anesthetics, are instrumented with needles and intravenous medications, and interact with multiple providers during the course of their hospitalization. There is also the inherent risk of catastrophic events associated with the percutaneous procedure, however small that risk may be. Given that "staged" percutaneous interventions are scheduled for a specific day, it is possible to monitor key health behaviors such as engagement in exercise and medication adherence in the weeks before and after the scheduled interventions. This monitoring is easily done objectively and unobtrusively using accelerometers to measure physical activity and electronic pill bottles (reviewed elsewhere in this issue) to measure medication adherence, thereby eliminating the problems inherent in self-report. Patterns of (non) adherence could then be observed in the days leading up to, and subsequent to the intervention, and the differential effects of this real life stressor on depressed vs. non-depressed post-ACS patients thereby determined. As with the three previously described potential biological mechanisms, the testing of this overall scenario - a comparison of depression and non-depressed patients after ACS, exposure to real world psychological stress, and examination of effects on critical 
health behaviors, represents a fourth vehicle for implementation of a Perfect Storm conceptualization.

\section{Summary}

Research on the determinants and mechanisms of recurrent ACS in patients with CHD has been hampered by a myopic focus on single risk factors or sets of risk factors that are assumed to each act independently. We propose a Perfect Storm conceptual model to advance an understanding of ASC recurrence within the field of behavioral cardiology. This framework proposes that the co-occurrence of multiple pathophysiologic and psychosocial factors, each acting in tandem with one another, offers a superior model by which to elucidate the risk of recurrent ACS in patients with CHD. We have presented an example of how the Perfect Storm framework can contribute to a deeper understanding of the mechanisms underlying the association of depression and risk of recurrent ACS and mortality by outlining a set of studies that will examine the confluence of depression and acute stress in the activation of four distinct pathways: thrombogenicity, coronary perfusion, arrhythmia, and behavior.

The Perfect Storm model has the potential to inform cutting edge programs of research in other areas of behavioral cardiology and within the field of cardiology more broadly by addressing the complex, interplay of the multiple processes involved in the progression of coronary atherosclerosis to recurrent ACS and mortality. It may ultimately inform the development and testing of novel primary and secondary prevention strategies, improve extant primary and secondary prevention strategies, and result in superior patient management. Indeed, a staggering number of American adults have CHD and remain at considerable risk for cardiovascular deterioration and recurrent ACS events. Although traditional conceptual models and research strategies have offset this risk somewhat, newer models and corresponding programs of research such as the Perfect Storm are urgently needed if progress is to continue.

\section{Acknowledgments}

This work was supported by grants HL-088117, HL-101663, HL-84034, HL084438, from the National Institutes of Health. The content is solely the responsibility of the author and does not necessarily represent the official views of the NIH.

\section{Abbreviations}

$\begin{array}{ll}\text { ACS } & \text { Acute Coronary Syndrome } \\ \text { BTG } & \beta \text { Thromboglobulin } \\ \text { CAD } & \text { Coronary Artery Disease } \\ \text { CFR } & \text { Coronary Flow Reserve } \\ \text { CHD } & \text { Coronary Heart Disease } \\ \text { ED } & \text { Emergency Department } \\ \text { ET-1 } & \text { Endothelin-1 } \\ \text { MI } & \text { Myocardial Infarction } \\ \text { MSI } & \text { Mental Stress Provoked Myocardial Ischemia } \\ \text { PF4 } & \text { Platelet factor 4 }\end{array}$




\section{References}

1. Naghavi M, Libby P, Falk E, et al. From vulnerable plaque to vulnerable patient: a call for new definitions and risk assessment strategies: Part I. Circulation. 2003; 108:1664-72. [PubMed: 14530185]

2. Naghavi M, Libby P, Falk E, et al. From vulnerable plaque to vulnerable patient: a call for new definitions and risk assessment strategies: Part II. Circulation. 2003; 108:1772-8. [PubMed: 14557340]

3. Arbab-Zadeh A, Nakano M, Virmani R, Fuster V. Acute coronary events. Circulation. 2012; 125:1147-56. [PubMed: 22392862]

4. Lloyd-Jones D, Adams RJ, et al. Writing Group Members. Heart disease and stroke statistics--2010 update: a report from the American Heart Association. Circulation. 2010; 121:e46-e215. [PubMed: 20019324]

5. Franco M, Cooper RS, Bilal U, Fuster V. Challenges and opportunities for cardiovascular disease prevention. Am J Med. 2011; 124:95-102. [PubMed: 21295188]

6. Roger VL, Go AS, Lloyd-Jones DM, et al. Executive summary: heart disease and stroke statistics--2012 update: a report from the American Heart Association. Circulation. 2012; 125:18897. [PubMed: 22215894]

7. Roger VL, Go AS, Lloyd-Jones DM, et al. Heart disease and stroke statistics--2011 update: a report from the American Heart Association. Circulation. 2011; 123:e18-e209. [PubMed: 21160056]

8. Davies MJ. The contribution of thrombosis to the clinical expression of coronary atherosclerosis. Thromb Res. 1996; 82:1-32. [PubMed: 8731506]

9. Loscalzo J, Kohane I, Barabasi AL. Human disease classification in the postgenomic era: a complex systems approach to human pathobiology. Mol Sys Biol. 2007; 3:124.

10. Van Regenmortel MH. Reductionism and complexity in molecular biology. Scientists now have the tools to unravel biological and overcome the limitations of reductionism. EMBO Rep. 2004; 5:1016-20. [PubMed: 15520799]

11. Bush DE, Ziegelstein RC, Tayback M, et al. Even minimal symptoms of depression increase mortality risk after acute myocardial infarction. Am J Cardiol. 2001; 88:337-41. [PubMed: 11545750]

12. Carney RM, Blumenthal JA, Catellier D, et al. Depression as a risk factor for mortality after acute myocardial infarction. Am J Cardiol. 2003; 92:1277-81. [PubMed: 14636903]

13. Lauzon C, Beck CA, Huynh T, et al. Depression and prognosis following hospital admission because of acute myocardial infarction. CMAJ. 2003; 168:547-52. [PubMed: 12615746]

14. Watkins LL, Schneiderman N, Blumenthal JA, et al. Cognitive and somatic symptoms of depression are associated with medical comorbidity in patients after acute myocardial infarction. Am Heart J. 2003; 146:48-54. [PubMed: 12851607]

15. Nicholson A, Kuper H, Hemingway H. Depression as an aetiologic and prognostic factor in coronary heart disease: a meta-analysis of 6362 events among 146538 participants in 54 observational studies. Eur Heart J. 2006; 27:2763-74. [PubMed: 17082208]

16. van Melle JP, de Jonge P, Spijkerman TA, et al. Prognostic association of depression following myocardial infarction with mortality and cardiovascular events: a meta-analysis. Psychosom Med. 2004; 66:814-22. [PubMed: 15564344]

17. Akhtar S, Husk G, Waxman D, Sonara P. Emergency department crowding: A national perspective. Ann Emerg Med. 2004; 44:S28.

18. Diercks DB, Roe MT, Chen AY, et al. Prolonged emergency department stays of non-ST-segmentelevation myocardial infarction patients are associated with worse adherence to the American College of Cardiology/American Heart Association guidelines for management and increased adverse events. Ann Emerg Med. 2007; 50:489-96. [PubMed: 17583379]

19. Handel DA, Pines J, Aronsky D, et al. Variations in crowding and ambulance diversion in nine emergency departments. Acad Emerg Med. 2011; 18:941-6. [PubMed: 21906203]

20. Pines JM, Pollack CV Jr, Diercks DB, Chang AM, Shofer FS, Hollander JE. The association between emergency department crowding and adverse cardiovascular outcomes in patients with chest pain. Acad Emerg Med. 2009; 16:617-25. [PubMed: 19549010] 
21. Wears RL. Heart bone connected to the... trauma bone? Ann Emerg Med. 2006; 48:355-7. [PubMed: 16997670]

22. Carney RM, Freedland KE, Miller GE, Jaffe AS. Depression as a risk factor for cardiac mortality and morbidity: a review of potential mechanisms. J Psychosom Res. 2002; 53:897-902. [PubMed: 12377300]

23. Carney RM, Freedland KE, Veith RC. Depression, the autonomic nervous system, and coronary heart disease. Psychosom Med. 2005; 67 (Suppl 1):S29-33. [PubMed: 15953797]

24. Gorman JM, Sloan RP. Heart rate variability in depressive and anxiety disorders. Am Heart J. 2000; 140:77-83. [PubMed: 11011352]

25. Musselman DL, Tomer A, Manatunga AK, et al. Exaggerated platelet reactivity in major depression. Am J Psychiatry. 1996; 153:1313-7. [PubMed: 8831440]

26. Shimbo D, Child J, Davidson K, et al. Exaggerated serotonin-mediated platelet reactivity as a possible link in depression and acute coronary syndromes. Am J Cardiol. 2002; 89:331-3. [PubMed: 11809437]

27. Burg MM, Martens EJ, Collins D, Soufer R. Depression predicts elevated endothelin-1 in patients with coronary artery disease. Psychosom Med. 2011; 73:2-6. [PubMed: 20947777]

28. Vaccarino V, Votaw J, Faber T, et al. Major depression and coronary flow reserve detected by positron emission tomography. Arch Intern Med. 2009; 169:1668-76. [PubMed: 19822823]

29. Ho PM, Spertus JA, Masoudi FA, et al. Impact of medication therapy discontinuation on mortality after myocardial infarction. Arch Intern Med. 2006; 166:1842-7. [PubMed: 17000940]

30. Rasmussen JN, Chong A, Alter DA. Relationship between adherence to evidence-based pharmacotherapy and long-term mortality after acute myocardial infarction. JAMA. 2007; 297:177-86. [PubMed: 17213401]

31. Kronish IM, Rieckmann N, Halm EA, et al. Persistent depression affects adherence to secondary prevention behaviors after acute coronary syndromes. J Gen Intern Med. 2006; 21:1178-83. [PubMed: 16899061]

32. Suls J, Bunde J. Anger, anxiety, and depression as risk factors for cardiovascular disease: the problems and implications of overlapping affective dispositions. Psychol Bull. 2005; 131:260 300. [PubMed: 15740422]

33. Burg MM, Lampert R, Joska T, Batsford W, Jain D. Psychological traits and emotion-triggering of ICD shock-terminated arrhythmias. Psychosom Med. 2004; 66:898-902. [PubMed: 15564355]

34. Musselman DL, Marzec UM, Manatunga A, et al. Platelet reactivity in depressed patients treated with paroxetine: preliminary findings. Arch Gen Psychiatry. 2000; 57:875-82. [PubMed: 10986551]

35. Laghrissi-Thode F, Wagner WR, Pollock BG, Johnson PC, Finkel MS. Elevated platelet factor 4 and beta-thromboglobulin plasma levels in depressed patients with ischemic heart disease. Biol Psychiatry. 1997; 42:290-5. [PubMed: 9270907]

36. Gehi A, Musselman D, Otte C, Bruce Royster E, Ali S, Whooley MA. Depression and platelet activation in outpatients with stable coronary heart disease: findings from the Heart and Soul Study. Psychiatry Res. 2010; 175:200-4. [PubMed: 20034674]

37. Serebruany VL, Glassman AH, Malinin AI, et al. Enhanced platelet/endothelial activation in depressed patients with acute coronary syndromes: evidence from recent clinical trials. Blood Coagul Fibrinolysis. 2003; 14:563-7. [PubMed: 12960610]

38. Thrall G, Lane D, Carroll D, Lip GY. A systematic review of the prothrombotic effects of an acute change in posture: a possible mechanism underlying the morning excess in cardiovascular events? Chest. 2007; 132:1337-47. [PubMed: 17934119]

39. Rajagopalan S, Brook R, Rubenfire M, Pitt E, Young E, Pitt B. Abnormal brachial artery flowmediated vasodilation in young adults with major depression. Am J Cardiol. 2001; 88:196-8. A7. [PubMed: 11448425]

40. Spieker LE, Hurlimann D, Ruschitzka F, et al. Mental stress induces prolonged endothelial dysfunction via endothelin-A receptors. Circulation. 2002; 105:2817-20. [PubMed: 12070106]

41. Schang SJ Jr, Pepine CJ. Transient asymptomatic S-T segment depression during daily activity. Am J Cardiol. 1977; 39:396-402. [PubMed: 402803] 
42. Burg MM, Soufer R. Mental stress-induced myocardial ischemia: moving forward. J Nucl Cardiol. 2007; 14:269-71. [PubMed: 17556157]

43. Jain D, Burg M, Soufer R, Zaret BL. Prognostic implications of mental stress-induced silent left ventricular dysfunction in patients with stable angina pectoris. Am J Cardiol. 1995; 76:31-5. [PubMed: 7793399]

44. Jiang W, Babyak M, Krantz DS, et al. Mental stress--induced myocardial ischemia and cardiac events. JAMA. 1996; 275:1651-6. [PubMed: 8637138]

45. Krantz DS, Santiago HT, Kop WJ, Bairey Merz CN, Rozanski A, Gottdiener JS. Prognostic value of mental stress testing in coronary artery disease. Am J Cardiol. 1999; 84:1292-7. [PubMed: 10614793]

46. Sheps DS, McMahon RP, Becker L, et al. Mental stress-induced ischemia and all-cause mortality in patients with coronary artery disease: Results from the Psychophysiological Investigations of Myocardial Ischemia study. Circulation. 2002; 105:1780-4. [PubMed: 11956119]

47. Yeung AC, Vekshtein VI, Krantz DS, et al. The effect of atherosclerosis on the vasomotor response of coronary arteries to mental stress. N Engl J Med. 1991; 325:1551-6. [PubMed: 1944439]

48. Arrighi JA, Burg M, Cohen IS, et al. Myocardial blood-flow response during mental stress in patients with coronary artery disease. Lancet. 2000; 356:310-1. [PubMed: 11071190]

49. Jiang W, Babyak MA, Rozanski A, et al. Depression and increased myocardial ischemic activity in patients with ischemic heart disease. Am Heart J. 2003; 146:55-61. [PubMed: 12851608]

50. Information on ED visits from the HCUP Nationwide Emergency Department Sample (NEDS). Agency for Healthcare Research and Quality; 2013. at http://hcupnet.ahrq.gov/HCUPnet.jsp? $\mathrm{Id}=\mathrm{C} 3960 \mathrm{D} 840 \mathrm{E} 5 \mathrm{E} 2205 \&$ Form $=$ SelPAT $\& \mathrm{JS}=\mathrm{Y} \&$ Action $=\% 3 \mathrm{E} \% 3 \mathrm{ENext} \% 3 \mathrm{E}$

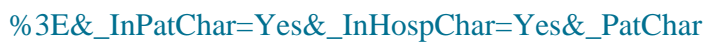

51. Hoot NR, Aronsky D. Systematic review of emergency department crowding: causes, effects, and solutions. Ann Emerg Med. 2008; 52:126-36. [PubMed: 18433933]

52. Edmondson D, Shimbo D, Ye S, Wyer P, Davidson KW. The Association of Emergency Department Crowding During Treatment for Acute Coronary Syndrome With Subsequent Posttraumatic Stress Disorder Symptoms. JAMA Intern Med. 2013:1-2.

53. Virtanen M, Pentti J, Vahtera J, et al. Overcrowding in hospital wards as a predictor of antidepressant treatment among hospital staff. Am J Psychiatry. 2008; 165:1482-6. [PubMed: 18676590]

54. Leor J, Poole WK, Kloner RA. Sudden cardiac death triggered by an earthquake. N Engl J Med. 1996; 334:413-9. [PubMed: 8552142]

55. Meisel SR, Kutz I, Dayan KI, et al. Effect of Iraqi missile war on incidence of acute myocardial infarction and sudden death in Israeli civilians. Lancet. 1991; 338:660-1. [PubMed: 1679475]

56. Steinberg JS, Arshad A, Kowalski M, et al. Increased incidence of life-threatening ventricular arrhythmias in implantable defibrillator patients after the World Trade Center attack. J Am Coll Cardiol. 2004; 44:1261-4. [PubMed: 15364329]

57. Lampert R, Joska T, Burg MM, Batsford WP, McPherson CA, Jain D. Emotional and physical precipitants of ventricular arrhythmia. Circulation. 2002; 106:1800-5. [PubMed: 12356633]

58. Stopper M, Joska T, Burg MM, et al. Electrophysiologic characteristics of anger-triggered arrhythmias. Heart Rhythm. 2007; 4:268-73. [PubMed: 17341385]

59. Lampert R, Shusterman V, Burg MM, et al. Effects of psychologic stress on repolarization and relationship to autonomic and hemodynamic factors. J Cardiovasc Electrophysiol. 2005; 16:372-7. [PubMed: 15828878]

60. Yip HK, Wu CJ, Chang HW, et al. Prognostic value of circulating levels of endothelin-1 in patients after acute myocardial infarction undergoing primary coronary angioplasty. Chest. 2005; 127:1491-7. [PubMed: 15888819]

61. Derlet RW, Richards JR. Ten solutions for emergency department crowding. West J Emerg Med. 2008; 9:24-7. [PubMed: 19561699]

62. Chow CK, Jolly S, Rao-Melacini P, Fox KA, Anand SS, Yusuf S. Association of diet, exercise, and smoking modification with risk of early cardiovascular events after acute coronary syndromes. Circulation. 2010; 121:750-8. [PubMed: 20124123] 
63. Rieckmann N, Burg MM, Kronish IM, Chaplin WF, Schwartz JE, Davidson KW. Aspirin adherence, depression and one-year prognosis after acute coronary syndrome. Psychother Psychosom. 2011; 80:316-8. [PubMed: 21720196]

64. Spertus JA, Kettelkamp R, Vance C, et al. Prevalence, predictors, and outcomes of premature discontinuation of thienopyridine therapy after drug-eluting stent placement: results from the PREMIER registry. Circulation. 2006; 113:2803-9. [PubMed: 16769908]

65. Bush, DE.; Ziegelstein, RC.; Patel, UV., et al. Evidence report/technology assessment (Summary). 2005. Post-myocardial infarction depression; p. 1-8.

66. Kronish IM, Rieckmann N, Shimbo D, Burg M, Davidson KW. Aspirin adherence, aspirin dosage, and C-reactive protein in the first 3 months after acute coronary syndrome. Am J Cardiol. 2010; 106:1090-4. [PubMed: 20920644]

67. Chapman RH, Benner JS, Petrilla AA, et al. Predictors of adherence with antihypertensive and lipid-lowering therapy. Arch Intern Med. 2005; 165:1147-52. [PubMed: 15911728]

68. Gazmararian JA, Kripalani S, Miller MJ, Echt KV, Ren J, Rask K. Factors associated with medication refill adherence in cardiovascular-related diseases: a focus on health literacy. J Gen Intern Med. 2006; 21:1215-21. [PubMed: 17105519]

69. Knafl GJ, Schoenthaler A, Ogedegbe G. Secondary analysis of electronically monitored medication adherence data for a cohort of hypertensive African-Americans. Patient Prefer adherence. 2012; 6:207-19. [PubMed: 22536057]

70. Modi AC, Rausch JR, Glauser TA. Patterns of nonadherence to antiepileptic drug therapy in children with newly diagnosed epilepsy. JAMA. 2011; 305:1669-76. [PubMed: 21521848]

71. Whooley MA, de Jonge P, Vittinghoff E, et al. Depressive symptoms, health behaviors, and risk of cardiovascular events in patients with coronary heart disease. JAMA. 2008; 300:2379-88. [PubMed: 19033588]

72. Grace SL, Abbey SE, Pinto R, Shnek ZM, Irvine J, Stewart DE. Longitudinal course of depressive symptomatology after a cardiac event: effects of gender and cardiac rehabilitation. Psychosom Med. 2005; 67:52-8. [PubMed: 15673624]

73. Motl RW, Birnbaum AS, Kubik MY, Dishman RK. Naturally occurring changes in physical activity are inversely related to depressive symptoms during early adolescence. Psychosom Med. 2004; 66:336-42. [PubMed: 15184692]

74. Lett HS, Blumenthal JA, Babyak MA, et al. Depression as a risk factor for coronary artery disease: evidence, mechanisms, and treatment. Psychosom Med. 2004; 66:305-15. [PubMed: 15184688]

75. Zoeller RF. Physical Activity: Depression, Anxiety, Physical Activity, and Cardiovascular Disease: What's the Connection? Am J Lifestyle Med. 2007; 1:175-80.

76. Blumenthal JA, Babyak MA, Moore KA, et al. Effects of exercise training on older patients with major depression. Arch Intern Med. 1999; 159:2349-56. [PubMed: 10547175]

77. Daley A. Exercise and depression: a review of reviews. J Clin Psychol Med Settings. 2008; 15:140-7. [PubMed: 19104978]

78. Oman RF, King AC. The effect of life events and exercise program format on the adoption and maintenance of exercise behavior. Health Psychol. 2000; 19:605-12. [PubMed: 11129364]

79. Bottonari KA, Safren SA, McQuaid JR, Hsiao CB, Roberts JE. A longitudinal investigation of the impact of life stress on HIV treatment adherence. J Behav Med. 2010; 33:486-95. [PubMed: 20577794]

80. Aldao A, Nolen-Hoeksema S, Schweizer S. Emotion-regulation strategies across psychopathology: A meta-analytic review. Clin Psychol Rev. 2010; 30:217-37. [PubMed: 20015584] 


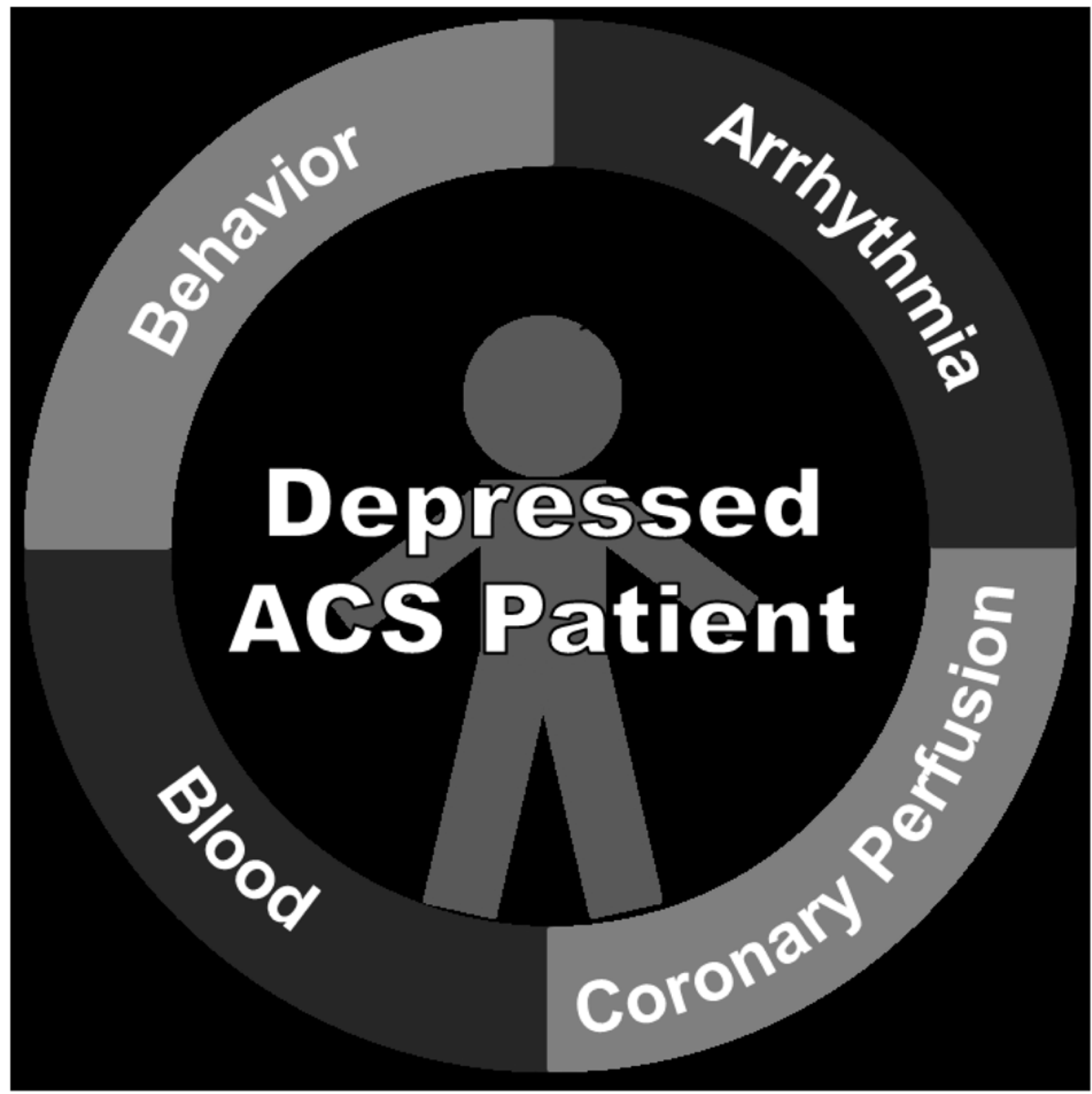

Figure 1.

A Perfect Storm Model of Depression and Post-ACS Recurrence/Mortality Risk 


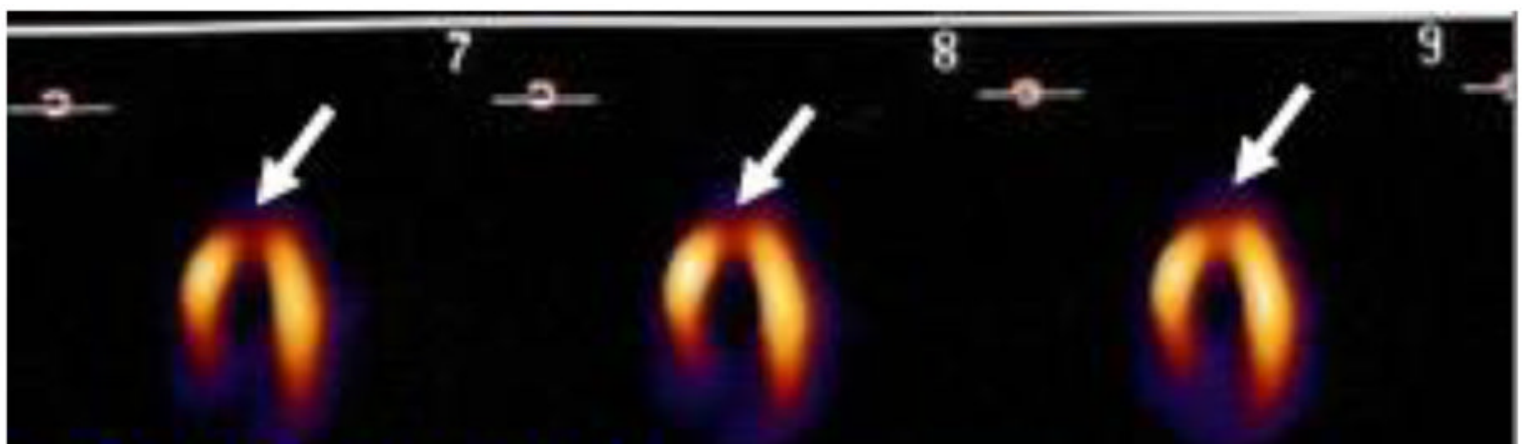

\section{Mental Stress Image}

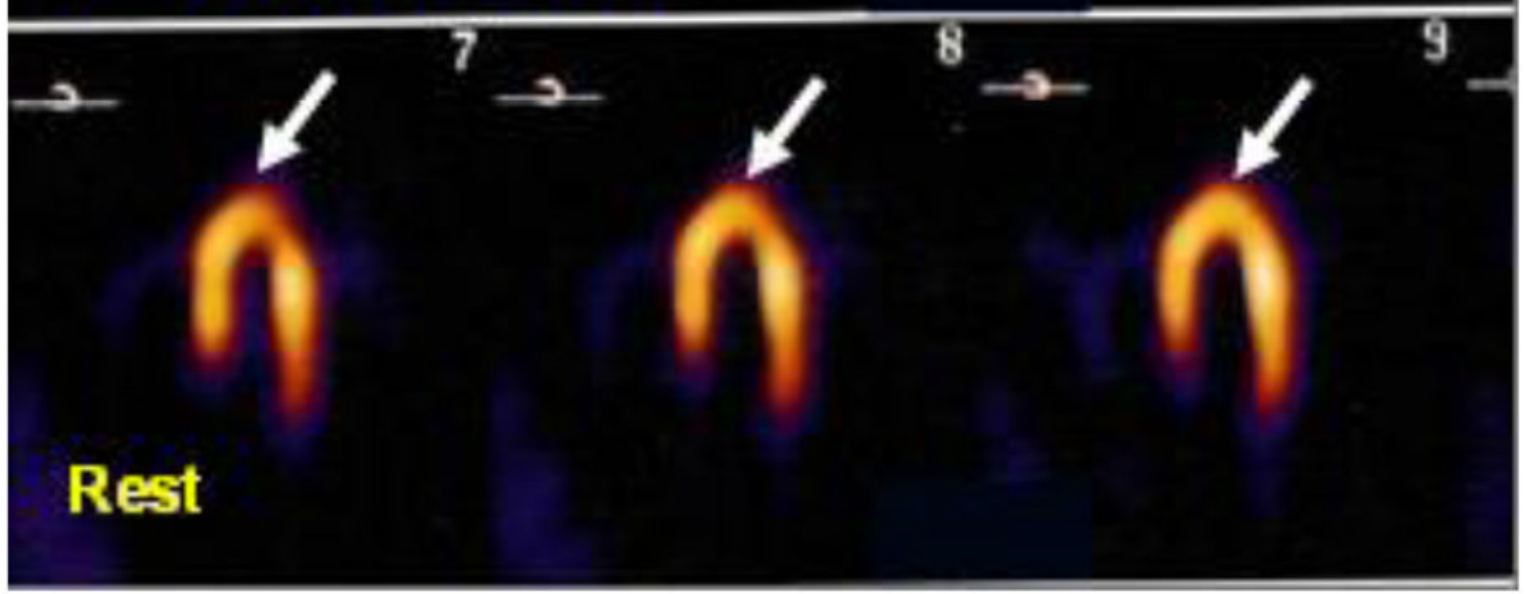

Figure 2.

Long Axis Myocardial Perfusion Image (MPI) demonstrating a new inferior/anterior myocardial perfusion defect with mental stress 


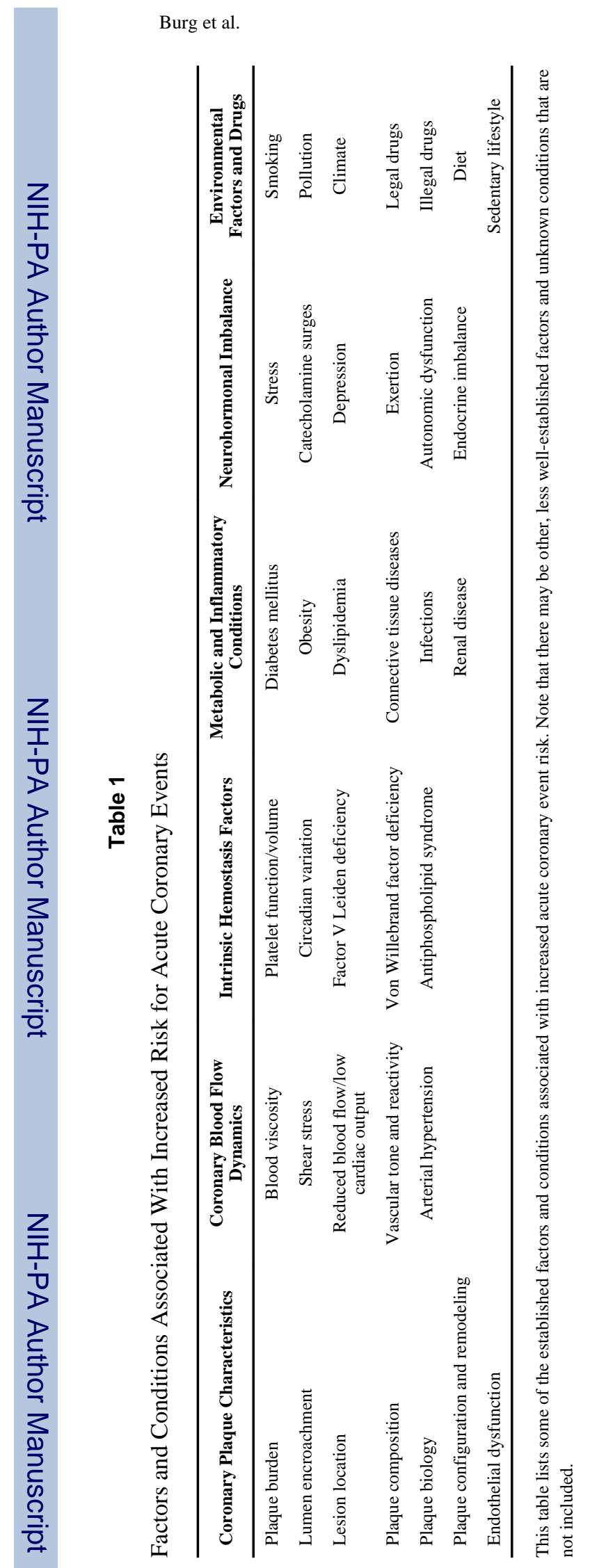

Page 17 